\title{
Evaluation of pulsed dose oxygen delivery during exercise in patients with severe chronic obstructive pulmonary disease
}

\author{
R Garrod, J C Bestall, E Paul, J A Wedzicha
}

\begin{abstract}
Background-Oxygen conserving devices may lead to substantial increases in the duration of oxygen provided. A study was undertaken to compare the performance of a pulsed dose oxygen delivery (PDOD) system with continuous flow oxygen or air during a maximal walking test.

Methods-Fourteen patients with chronic obstructive pulmonary disease (COPD) and arterial oxygen desaturation on exercise (mean (SD) forced expiratory volume in one second $\left(\mathrm{FEV}_{1}\right) 0.83(0.28) \mathbf{1}$, arterial oxygen pressure $\left(\mathrm{PaO}_{2}\right) 8.38$ (1.24) kPa, arterial carbon dioxide pressure $\left(\mathrm{PaCO}_{2}\right)$ $5.95(0.86) \mathrm{kPa})$ were randomised to perform a walking test using air administered via a cylinder or continuous flow oxygen at $21 /$ min or by a PDOD system.

Results-There was no significant difference in the mean arterial oxygen saturation $\left(\mathrm{SaO}_{2}\right)$ using the PDOD system or with continuous flow oxygen $(p=0.33)$. Patients showed greatest desaturation whilst walking with the air cylinder $\left(\mathrm{SaO}_{2}\right.$ $79.2(8.59) \%)$ which was significantly different from the desaturation with both continuous flow oxygen $(87.6(5.85) \%, p=$ $0.001)$ and PDOD (85.6 (7.36)\%, p = $0.004)$. There was no significant difference between the distance walked using oxygen delivered at $21 / \mathrm{min}$ by continuous flow or via the PDOD ( $p=0.72$; CI 0.34 to 1.08 ). The mean (SD) distance walked on continuous flow oxygen $(203.6(106.1) \mathrm{m})$ and PDOD (207.9 (109.8) m) was significantly greater than the distance walked with the air cylinder $(188.6(110.02) \mathrm{m})$; (1.12 fold increase in distance, $\mathrm{CI} 1.01$ to $1.23, \mathrm{p}=$ 0.02 , and 1.14 fold increase in distance, CI 1.01 to $1.28, \mathrm{p}=0.03$, respectively).

Conclusions-These findings suggest that the pulsed dose oxygen conserving device was as effective as continuous flow oxygen in maintaining arterial oxygen saturation and that the use of this device was associated with similar improvements in exercise tolerance to patients taking continuous flow oxygen therapy.

(Thorax 1999;54:242-244)
\end{abstract}

Keywords: chronic obstructive pulmonary disease; exercise; oxygen therapy; pulsed dose oxygen delivery

Studies have shown that the use of supplemental oxygen during exercise may reduce dyspnoea and improve exercise performance for patients with severe chronic obstructive pulmonary disease (COPD) and hypoxaemia. ${ }^{1-3}$ However, continuous flow oxygen is wasteful and provides only a relatively short duration of oxygen. The recent development of oxygen conserving devices allows substantial increases in the duration of oxygen provided. ${ }^{45}$ Although these devices are effective at rest, there have been few studies of their performance during exercise.

One study which evaluated a number of oxygen conserving devices during exercise showed that devices capable of increasing the amount of oxygen with increasing respiratory rates performed better than those in which delivery of oxygen did not alter with respiratory rate. ${ }^{6}$ Another study which investigated the effectiveness of an oxygen conserving device that delivers oxygen only on intermittent breaths showed a fall in arterial oxygen saturation during exercise. ${ }^{7}$

The purpose of this study was to evaluate the effectiveness of a newly developed pulsed dose oxygen delivery (PDOD) system during exercise in patients with severe COPD. The PDOD device delivers a bolus of oxygen at the start of inspiration, thereby maximising therapeutic benefit during exercise as respiratory rates increase. This may lead to improved oxygenation during exercise when compared with continuous flow oxygen delivery.

\section{Methods}

Fifteen patients with stable COPD were referred from the outpatient clinics of the London Chest Hospital. Entry criteria included forced expiratory volume in one second $\left(\mathrm{FEV}_{1}\right)$ of less than one litre, less than $15 \%$ and $200 \mathrm{ml}$ reversibility with $\beta$ agonists, and no exacerbation during the previous four weeks. Patients had arterial oxygen desaturation $\left(\mathrm{SaO}_{2}\right)$ of at least $4 \%$ on a baseline walk.

Resting blood gas tensions in ear lobe samples obtained while breathing room air at rest for at least 20 minutes were measured 
using a Ciba-Corning 278 blood gas analyser (Medfield, Massachusetts, USA) ${ }^{8}$ and spirometric tests were performed using a rolling seal spirometer (P K Morgan Ltd, Rainham, UK).

The study was approved by the ethics committee of East London and the City Health Authority with all patients providing informed consent.

EXERCISE PROTOCOL

Exercise capacity was assessed using the shuttle walk test (SWT) which is a maximal externally paced incremental exercise test, ${ }^{9}$ together with the Borg dyspnoea score before and immediately after each walking test. ${ }^{10} \mathrm{SaO}_{2}$ was monitored throughout the walk using a pulse oximeter (Minolta Pulsox 7; AVL Instruments, Schaffhausen, Switzerland) and the lowest $\mathrm{SaO}_{2}$ value was recorded.

Patients performed a baseline walk on room air and were then randomly assigned to three interventions on separate occasions. The codes for randomisation were held in sealed envelopes.

(1) Continuous flow oxygen provided via nasal cannulae at a flow rate of $21 / \mathrm{min}$ from a portable 230 litre cylinder (Sabre, Aldershot, UK). The duration of supply was about 115 minutes and the weight $2.9 \mathrm{~kg}$.

(2) Air provided from an identical cylinder designed to be used as a placebo in controlled trials. The delivery of air was also via nasal cannulae at $2 \mathrm{l} / \mathrm{min}$.

(3) Demand flow oxygen supplied by an identical portable oxygen cylinder fitted with a Pulsedose EX 2000 battery powered oxygen conserving device (DeVilbiss Healthcare, Stourbridge, UK) with an additional weight of $0.87 \mathrm{~kg}$, also set to deliver oxygen at a flow rate equivalent to $2 \mathrm{l} / \mathrm{min}$.

The Pulsedose EX 2000 unit operates by delivering a bolus (pulse) of oxygen at the beginning of each breath. A pressure sensor sends a signal to the circuit board as soon as a negative pressure is detected in the cannulae. The valve is then turned on for a set length of time, depending upon the flow setting. The time for each flow setting ranges from 0.05 seconds to 0.6 seconds and oxygen flows at $10 \mathrm{l} / \mathrm{min}$ during the time the valve is open, providing oxygen flow rates equivalent to 2,4 , or $6 \mathrm{l} / \mathrm{min}$.

All patients carried the cylinders in a shoulder bag and were blinded as to whether they were breathing oxygen or air for interventions 1 and 2. It was not possible to blind the researcher to the contents of the cylinder or to the use of PDOD. Patients were instructed to breathe nasally on all walks and to stop walking when no longer able to maintain the required speed or to continue further.

STATISTICAL ANALYSIS

After log transformation the walking distance was normally distributed and the paired $t$ test was used to compare log walk on air, oxygen, and PDOD. $\mathrm{SaO}_{2}$ values could not be transformed to a normal distribution so the equivalent non-parametric Wilcoxon signed ranks were used. For both walking distance and $\mathrm{SaO}_{2}$

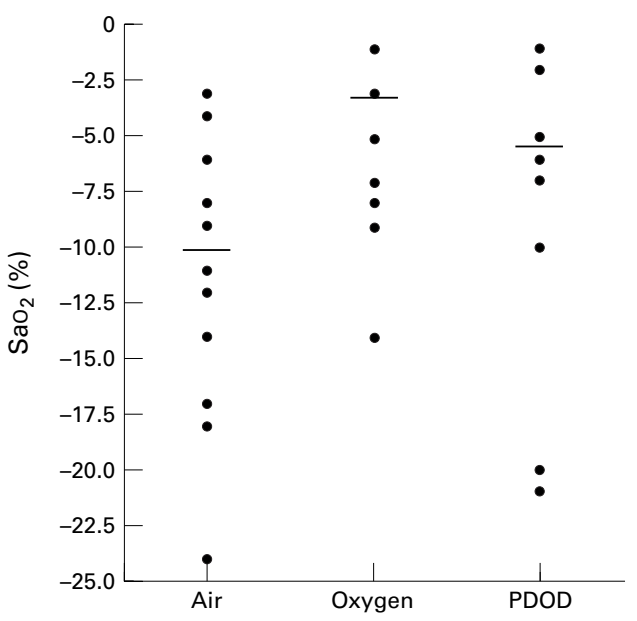

Figure 1 Changes in arterial saturation during shuttle walk test whilst breathing air, continuous flow oxygen, and oxygen by a pulsed dose delivery system (PDOD). Horizontal bars represent medians.

the changes between different treatment modes were calculated and the medians and interquartile ranges of these changes are given.

\section{Results}

Patients had a mean age (range) of 66 (50-75) years, mean (SD) $\mathrm{FEV}_{1}$ of $0.83(0.28) 1$ ( $\%$ predicted $32(9.40) \%), \mathrm{PaO}_{2} 8.38(1.24) \mathrm{kPa}$, and $\mathrm{PaCO}_{2} 5.95(0.86) \mathrm{kPa}$. One patient was excluded as she was unable to complete the walk because of a panic attack caused by an inability to breathe nasally.

\section{EXERCISE ARTERIAL SATURATION}

Patients showed the lowest $\mathrm{SaO}_{2}$ after walking while breathing air (median $78 \%$ ) which was significantly different from the minimum $\mathrm{SaO}_{2}$ on either continuous flow oxygen $(88.5 \%, \mathrm{p}=$ $0.001)$ or PDOD $(87 \%, p=0.004)$. There was no significant difference between the lowest $\mathrm{SaO}_{2}$ whilst breathing oxygen via the PDOD device or breathing continuous flow oxygen ( $p$ $=0.33$ ).

The median change in $\mathrm{SaO}_{2}$ on oxygen was $3 \%$ (interquartile range (IQR) $0-7$ ), on PDOD was $5.5 \%$ (IQR 7-5), and on air was $10 \%$ (IQR 4-17) (fig 1).

\section{EXERCISE TOLERANCE}

There were no statistical differences in dyspnoea as assessed by the Borg score, regardless of delivery device.

The mean (SD) baseline walks on air, continuous flow oxygen, and PDOD were $188.6(110.0) \mathrm{m}, 203.6$ (106.1) $\mathrm{m}$, and 207.9 (109.8) m, respectively.

There was a significant difference in the log walking distance with continuous flow oxygen compared with air (1.12 fold increase, CI 1.01 to $1.23 ; \mathrm{p}=0.028)$. Similarly, there was a significant difference in log walking distance with PDOD compared with air (1.14 fold increase, CI 1.01 to $1.28 ; \mathrm{p}=0.03)$. There was no significant difference in the walking distance with continuous flow oxygen and PDOD. 


\section{Discussion}

This study shows that the pulsed dose oxygen conserving device was as effective as continuous flow oxygen in maintaining arterial oxygen saturation on exercise in patients with severe COPD. The use of this device was associated with similar improvements in exercise tolerance to patients taking continuous flow oxygen therapy. It is possible that, if a submaximal exercise test were used rather than a maximal walking test, the differences between walking with oxygen and air may have been even greater. ${ }^{11}$

These findings are in contrast to the study by Roberts and colleagues who investigated an oxygen conserving device that delivers only intermittent boluses of oxygen. ${ }^{7}$ The difference in effectiveness of these two types of oxygen conserving devices is likely to be due to differences in triggering. The intermittent device delivers a bolus of oxygen every second breath at an equivalent rate of $21 / \mathrm{min}$ while the PDOD delivers oxygen with every breath. Thus the PDOD is particularly advantageous for patients with higher respiratory rates on exercise. In some of our patients the inspired flow rate of $21 / \mathrm{min}$ was insufficient to maintain adequate oxygen saturation during exercise, even with continuous flow. Those patients who continued to have oxygen desaturation whilst breathing continuous flow oxygen showed similar effects whilst breathing oxygen via PDOD. The flow rate of $2 \mathrm{l} / \mathrm{min}$ was chosen for this study for purposes of standardisation, although higher flow rates could have been more effective in correcting exercise desaturation and improving exercise tolerance.

The addition of oxygen conserving devices to portable cylinders or liquid oxygen tanks can prolong the time of oxygen delivery. In this study we found that oxygen provided by PDOD lasted four times longer than continuous flow oxygen, with the PDOD system costing an extra $£ 410$. The use of oxygen conserv- ing devices with portable gaseous oxygen cylinders may have considerable economic advantages over liquid oxygen and continuous flow systems when the extra duration of provision is considered. However, the type of device used requires consideration and oxygen conserving devices that provide a bolus of oxygen with each breath have advantages over intermittent oxygen supplementation, especially for patients with severe COPD and exercise desaturation. If these devices are provided for patients, careful assessment is required with measurement of arterial oxygen saturation on an exercise test. Adjustment of the oxygen flow rate, in combination with the device used, is required to ensure optimum benefit and effectiveness.

We acknowledge DeVilbiss Healthcare Ltd for the loan of the Pulsedose EX2000 oxygen conservation device. R Garrod and J Bestall were funded by the UK National Health Service Research and Development Programme.

1 Davidson AC, Leach R, George RJD, et al. Supplemental oxygen and exercise ability in chronic obstructive pulmonary disease. Thorax 1988;43:965-71.

2 Waterhouse J, Howard P. Breathlessness and portable oxygen in chronic airways disease. Thorax 1983;38:302-6.

3 Lock SH, Paul EA, Rudd RM, et al. Portable oxygen therapy: assessment and usage. Respir Med 1991;85:40712.

4 Tiep L, Carter R, Nicotra B, et al. Demand oxygen delivery during exercise. Chest 1987;91:15-20.

5 Bower JS, Brook C, Zimmer K, et al. Performance of a demand oxygen saver system during rest and sleep in demand oxygen saver system during rest

6 Braun SR, Spratt G, Scott GC, et al. Comparison of six oxyBraun SR, Spratt G, Scott GC, et al. Comparison of six oxy-
gen delivery systems for COPD patients at rest and during gen delivery systems for COPD
exercise. Chest 1992;102:694-8.

7 Roberts CM, Bell J, Wedzicha JA. Comparison of the efficacy of a demand oxygen delivery system with continuous low flow oxygen in subjects with stable COPD and severe oygen desaturation on walking. Thorax 1996;51: 831-4.

8 Pitkin AD, Roberts CM, Wedzicha JA. Arterialised ear lobe blood gas analysis: an underused technique. Thorax 1994;49:364-6.

9 Singh SJ, Morgan MDL, Scott S, et al. Development of a shuttle walking test of disability in patients with chronic airways obstruction. Thorax 1991;47:1019-24.

10 Burdon GW, Juniper EF, Killian KJ, et al. The perception of breathlessness in asthma. Am Rev Respir Dis 1982;126:8258.

11 Bradley BL, Garner AE, Billiu D, et al. Oxygen-assisted exercise in chronic obstructive lung disease. Am Rev Respir Dis 1978;118:239-43. 